International Mathematical Forum, 2, 2007, no. 25, 1221 - 1223

\title{
Pointed Nodal Plane Curves with the Expected Number of Moduli
}

\author{
E. Ballico ${ }^{1}$ \\ Dept. of Mathematics \\ University of Trento \\ 38050 Povo (TN), Italy \\ ballico@science.unitn.it
}

\begin{abstract}
Here we describe families of plane curves passing through $s$ fixed points and with the expect number of moduli as $s$-pointed curves.

Mathematics Subject Classification: 14H50; $14 \mathrm{H} 51$
\end{abstract}

Keywords: pointed curve; plane nodal curve; moduli of pointed curves; gonality

For all integers $g, s$ such that either $g \geq 2$ and $s \geq 0$ or $g=1$ and $s \geq 1$ or $g=0$ and $s \geq 3$ let $\mathcal{M}_{g, s}$ denote the moduli space of all pairs $\left(C,\left(P_{1}, \ldots, P_{s}\right)\right)$, where $C$ is a smooth genus $g$ curve and $\left(P_{1}, \ldots, P_{s}\right)$ is an ordered set of $s$ distinct points of $C$. From now on we assume $g \geq 2$. Let $A$ be a smooth and connected projective surface and $\Gamma$ a locally closed subset of $\operatorname{Hilb}(A)$ such the normalization of every $T \in \Gamma$ is a smooth genus $g$ curve. Fix $s$ general points $Q_{1}, \ldots, Q_{s} \in A$ and set $\Gamma\left(Q_{1}, \ldots, Q_{s}\right):=\left\{T \in \Gamma: Q_{i} \in T_{\text {reg }}\right.$ for all $i\}$. For any $T \in \Gamma\left(Q_{1}, \ldots, Q_{s}\right)$ the normalization map $\phi: C \rightarrow T$ induces a a pointed curve $\left(C, \phi^{-1}\left(Q_{1}\right), \ldots, \phi^{-1}\left(Q_{s}\right)\right)$. Hence for a fixed choice of the points $Q_{1}, \ldots, Q_{s}$ the set $\Gamma$ induces a morphism $\eta_{s}: \Gamma\left(Q_{1}, \ldots, Q_{s}\right) \rightarrow \mathcal{M}_{g, s}$. We are interested in the integer $\operatorname{dim}\left(\operatorname{Im}\left(\eta_{s}\right)\right)$, i.e. in the number of moduli of the family $\Gamma\left(Q_{1}, \ldots, Q_{s}\right)$, mainly when $A=\mathbf{P}^{2}$. In the next statement for any non-empty algebraic set $W$ the integer $\operatorname{dim}(W)$ will denote the maximal dimension of one of its irreducible components. In the statements and proofs of Theorems 1 and 2 we will only apply this convenction when $W$ is equidimensional. We will first prove the following result.

Proposition 1. Let $A$ be a smooth and connected projective surface and $\Gamma$ a non-empty locally closed subset of $\operatorname{Hilb}(A)$ such the normalization of every $T \in$

\footnotetext{
${ }^{1}$ The author was partially supported by MIUR and GNSAGA of INdAM (Italy).
} 
$\Gamma$ is a smooth genus $g \geq 2$ curve. Fix a positive integer $s$ and $s$ general points $Q_{1}, \ldots, Q_{s} \in A$. Set $\epsilon_{0}:=\operatorname{dim}(\operatorname{Aut}(A)), I_{s}:=\left\{h \in \operatorname{Aut}(A): h\left(Q_{i}\right)=Q_{i}\right)$ for all $i\}$ and $\epsilon_{s}:=\operatorname{dim}\left(I_{s}\right)$. Assume that $\Gamma$ has the expected number of moduli, i.e. that the normalization map induces a morphism $\eta_{0}: \Gamma \in \mathcal{M}_{g}$ such that $\operatorname{dim}\left(\operatorname{Im}\left(\eta_{0}\right)\right)=\min \left\{3 g-3, \operatorname{dim}(\Gamma)-\epsilon_{0}\right\}$. Then $\Gamma\left(Q_{1}, \ldots, Q_{s}\right)$ has the expected number of moduli, i.e.

(i) $\Gamma\left(Q_{1}, \ldots, Q_{s}\right)=\emptyset$ if $\operatorname{dim}(\Gamma)<s$;

(ii) $\operatorname{dim}\left(\Gamma\left(Q_{1}, \ldots, Q_{s}\right)\right)=\operatorname{dim}(\Gamma)-s$ and $\operatorname{dim}\left(\operatorname{Im}\left(\eta_{s}\right)\right)=\min \{3 g-3+$ $\left.s, \operatorname{dim}(\Gamma)-s-\epsilon_{s}\right\}$ if $\operatorname{dim}(\Gamma) \geq s$.

The following result is an easy consequence of Proposition 1 and part (3) of [3], Th. 4.2.

Theorem 1. Fix integers $d, g, s$ such that $g \geq 2, s \geq 0$ and $(d-1)(d-2) / 2 \geq$ g. Fix s general points $Q_{1}, \ldots, Q_{s} \in \mathbf{P}^{2}$ and let $M$ be the family of all integral degree d curves $T \subset \mathbf{P}^{2}$ such that $T$ has exactly $(d-1)(d-2) / 2-g$ ordinary nodes as its only singularities and $Q_{i} \in T_{\text {reg }}$ for all $i$. Set $\epsilon_{s}=8-2 s$ if $s \leq 3$ and $\epsilon_{s}=0$ if $s \geq 4$. Then $M$ has the expected number of moduli, i.e. $M=\emptyset$ if $s \geq g+3 d, \operatorname{dim}(M)=g-s+3 d-1$ if $s \leq g+3 d-1$ and $\operatorname{dim}\left(\operatorname{Im}\left(\eta_{s}\right)\right)=\min \left\{3 g-3+s, g-s+3 d-1-\epsilon_{s}\right\}$ if $s \leq g+3 d-1$.

We need the integer $\epsilon_{s}$ because $\operatorname{dim}\left(\operatorname{Aut}\left(\mathbf{P}^{2}\right)=8\right.$ and any 4 points of $\mathbf{P}^{2}$, no 3 of them collinear, are projectively equivalent.

Then we will consider pointed $k$-gonal curve. For all integers $g, k$ such that $g \geq 2$ and $k \geq 2$ set $\mathcal{M}_{g}^{1, k}:=\left\{C \in \mathcal{M}_{g}: C\right.$ has a $\left.g_{k}^{1}\right\}$. Hence $\mathcal{M}_{g}^{1, k}=\mathcal{M}_{g}$ if $g \leq 2 k-2$, while $\mathcal{M}_{g}^{1, k}$ is an integral $(2 g+2 k-5)$-dimensional variety if $g \geq 2 k-3$. The following result is an easy consequence of Proposition 1 and [1], Th. 0.2.

Theorem 2. Fix integers $g, d, k, s$ such that $g \geq 2 k-3 \geq 1, s \geq 0$ and $d \geq(g+k+2) / 2$. Fix $s+1$ general points $Q_{0}, \ldots, Q_{s}$ in $\mathbf{P}^{2}$ and let $M_{s}$ denote the set of all integral degree $d$ curve $T \subset \mathbf{P}^{2}$ such $Q_{i} \in T_{\text {reg }}$ for $1 \leq$ $i \leq s, Q_{0}$ is an ordinary point with multiplicity $d-k$ of $T$ and $T$ has further $(d-2)(d-1) / 2-g-(d-k)(d-k-1) / 2$ ordinary nodes as its only singularities. Set $\epsilon_{s}=6-2 s$ if $s \leq 2$ and $\epsilon_{s}=0$ if $s \geq 3$.

(i) $M_{s}=\emptyset$ if $s \geq 4 d-k+g$;

(ii) Assume $s \leq 4 d-k+g-1$. Then $\operatorname{dim}\left(M_{s}\right)=4 d-k+g-1-s$ and $\operatorname{dim}\left(\operatorname{Im}\left(\eta_{s}\right)\right)=\min \left\{2 g+2 k-5,4 d-k+g-1-s-\epsilon_{s}\right\}$. 0 .

To use [1] and [3] we work over an algebraically closed field $\mathbb{K}$ with char $(\mathbb{K})=$

Proof of Proposition 1. We will use induction on $s$, the beginning case of the induction being the case $s=0$ which is true by assumption. Assume $s>0$ and that the result is true for the integer $s^{\prime}:=s-1$. Fix any irreducible component $M$ of $\Gamma\left(Q_{1}, \ldots, Q_{s-1}\right.$ and set $M\left(Q_{s}\right):=\left\{T \in M: Q_{s} \in T_{\text {reg }}\right\}$. We fix $Q_{1}, \ldots, Q_{s-1}$ and one of the finitely many $M$ 's and then move $Q_{s}$. We get 
$M\left(Q_{s}\right)=\emptyset$ if $\operatorname{dim}(M)=0$ and $\operatorname{dim}\left(M\left(Q_{s}\right)\right)=\operatorname{dim}(M)-1$ if $\operatorname{dim}(M)>0$. Hence $\Gamma\left(Q_{1}, \ldots, Q_{s}\right)=\emptyset$ if $\operatorname{dim}(\Gamma)<s$, while $\operatorname{dim}\left(\Gamma\left(Q_{1}, \ldots, Q_{s}\right)\right)=\operatorname{dim}(\Gamma)-$ $s$ if $\operatorname{dim}(\Gamma) \geq s$. We only need to prove the second assertion of part (ii). Let $A_{i}, I \in I$, with $I$ a finite set, the irreducible components of the general fiber of $\eta_{s-1}$ over any fixed irreducible component of $\operatorname{Im}\left(\eta_{s-1}\right)$. We have fixed $Q_{1}, \ldots, Q_{s-1}$ and then move $Q_{s}$. For every $i \in I$ we get $A_{i} \cap \Gamma\left(Q_{1}, \ldots, Q_{s}\right)=\emptyset$ if $\operatorname{dim}\left(A_{i}\right)=0$ and $\operatorname{dim}\left(A_{i} \cap \Gamma\left(Q_{1}, \ldots, Q_{s}\right)\right) \leq \operatorname{dim}\left(A_{i}\right)-1$ if $\operatorname{dim}(A)>0$. We get $\operatorname{dim}\left(\operatorname{Im}\left(\eta_{s}\right)\right) \geq \min \{3 g-3+s, \operatorname{dim}(\Gamma)-s\}$ if $\operatorname{dim}(\Gamma) \geq s$. Since the reverse inequality is obvious, we are done.

Proof of Theorem 1. If $s=0$, then $M$ is irreducible ([2]). Hence the case $s=0$ is part (3) of [3], Th. 4.2. If $s>0$, then apply Proposition 1.

Proof of Theorem 2. Let $u: A \rightarrow \mathbf{P}^{2}$ be the blowing-up of $Q_{0}$. Hence $A \cong F_{1}$ and $\operatorname{Pic}(A) \cong \mathbb{Z}^{2}$. Set $Q_{i}^{\prime}:=u^{-1}\left(Q_{i}\right)$ for $1 \leq i \leq s$. We will take $h:=u^{-1}\left(Q_{0}\right)$ and a fiber $f$ of a ruling of $A$ as a basis of $\operatorname{Pic}(A)$. Hence $h^{2}=-1, h \cdot f=1$ and $f^{2}=0$. For any integral curve $T \in \mathbf{P}^{2}$ let $T^{\prime}$ denote the strict transform of $T$ in $u$. $T \in M_{0}$ if and only if $T^{\prime}$ is a nodal integral element of $|k h+(d+k) f|$ with geometric genus $g$ and intersecting transversally $h$. Conversely, if $E \in|k h+(d+k) f|$ is a nodal integral curve with geometric genus $g$ and intersecting transversally $h$, then $u(E) \in M_{0}$. Let $W_{g}$ denote the set of all integral nodal curves $E \in|k h+(d+k) f|$ with geometric genus g. Apply [4], Cor 2.14, to a general union of $k$ general smooth (and hence rational) members of $h+f$ and $d-k$ general elements of $|f|$. You get $W_{g} \neq \emptyset$ and $\operatorname{dim}\left(W_{g}\right)=4 d-k+g-1$. $W_{g}$ is irreducible $([5])$. Hence $M_{0}$ is irreducible and $\operatorname{dim}\left(M_{0}\right)=4 d-k+g-1$. By Proposition 1 it is sufficient to prove the case $s=0$. Since $d \geq(g+k+2) / 2$, Theorem 2 is true by [1], Th. 0.2 .

\section{REFERENCES}

[1] E. Arbarello and M. Cornalba, Footnotes to a paper of Beniamino Segre, Math. Ann. 256 (1981), no. 3, 341-362.

[2] J. Harris, On the Severi problem, Invent. Math. 84 (1986), no. 3, 445-461.

[3] E. Sernesi, On the existence of certain families of curves, Invent. Math. 75 (1984), no. $1,25-57$.

[4] A. Tannenbaum, Families of algebraic curves with nodes, Compositio Math. 41 (1980), no. $1,107-126$.

[5] I. Tyomkin, On Severi varieties of Hirzebruch surfaces, e-print arXiv math.AG/0607807.

\section{Received: September 20, 2006}

